\title{
Early activation of the interleukin-23-17 axis in a murine model of oropharyngeal candidiasis
}

Type:

Article

Abstract:

$\mathrm{P}>$ Candida albicans is an oral commensal yeast that causes oropharyngeal candidiasis (OPC) in immunocompromised individuals. The immunological pathways involved in OPC have been revisited after the interleukin-17 (IL-17) pathway was implicated in fungal immunity. We studied immediate $(<24 \mathrm{~h}$ ) and adaptive (3-6 day) IL-12 and IL-2317 pathway activation in naive p40-/- mice, which lack IL-12 and IL-23 and develop severe, chronic OPC upon oral inoculation with C. albicans. Macrophages from p40-/mice were less efficient than $\mathrm{C} 57 \mathrm{BL} / 6 \mathrm{~J}$ controls at killing $\mathrm{C}$. albicans in vitro but very low numbers in the oral mucosae of infected C57BL/6J mice suggest that they are not critical in vivo, at least in this strain. Migration of macrophages to regional lymph nodes of infected p40-/- mice was impaired; however, dendritic cell migration was not affected. Recombinant IL-12 therapy provided only temporary relief from OPC, suggesting that IL-23 is required for full protection. In C57BL/6J mice, but not p40-/- mice, messenger RNAs encoding IL-23p19 and IL-17 were induced in the oral mucosa within $24 \mathrm{~h}$ of infection (6 +/- 0.6 and $12+/-2.7$-fold). By day 6 of infection in C57BL/6J mice, IL-17A messenger RNA level had increased $5.1+/-1.8$ and $83+/-21$-fold in regional lymph nodes and oral tissues respectively. Ablation of p40 was associated with delayed or abrogated induction of IL-17A pathway targets (monocyte chemoattractant protein-1, IL6 and macrophage inflammatory protein-2), and a lack of organized recruitment of neutrophils to the infected oral mucosa. Overall our data show that the IL-23-17A axis is activated early in the oral mucosae of immunologically naive mice with OPC.

\begin{tabular}{|c|l|}
\hline Author & $:$ Saunus, J. M. \\
& $\quad$ Wagner, S. A. \\
& $:$ Matias, M. A. \\
& $:$ Hu, Y. \\
& $\quad$ Zaini, Z. M. \\
& \\
\hline Source & Molecular Oral C. S. \\
\hline ISSN & $2041-1006$ \\
\hline DOI & - \\
\hline Volume (Issue) & $25(5)$ \\
\hline Page & $343-356$ \\
\hline Year & 2010 \\
\hline
\end{tabular}


Keyword:

Candida albicans, interleukin 23,interleukin 17A,oropharyngeal,candidiasis delta t-cells, human dendritic cells, ifn-gamma-production, hyper-ige,syndrome, in-vitro adaptive immunity,oral candidiasis,il-17,production,th17 cells, cryptococcus-neoformans

Please Cite As:

SAUNUS, J. M., WAGNER, S. A., MATIAS, M. A., HU, Y., ZAINI, Z. M. \& FARAH, C. S. 2010. Early activation of the interleukin-23-17 axis in a murine model of oropharyngeal candidiasis. Molecular Oral Microbiology, 25, 343-356.

URL:

- http://apps.webofknowledge.com search via Accession No >>000281551700004

- http://www.ncbi.nlm.nih.gov/pubmed/20883223 